\title{
Probiotic lactobacilli mediated changes in global epigenetic signatures of human intestinal epithelial cells during Escherichia coli challenge
}

\author{
Mohd Iqbal Bhat ${ }^{1} \cdot$ Ankita Kumari $^{1} \cdot$ Suman Kapila ${ }^{1} \cdot$ Rajeev Kapila ${ }^{1}$ \\ Received: 26 October 2018 / Accepted: 11 February 2019 / Published online: 26 February 2019 \\ (C) Università degli studi di Milano 2019
}

\begin{abstract}
Host genome environment association is critical for proper development and functioning of an individual. Microbiota and probiotics within the cellular vicinity may serve such critical stimuli that can bring out different epigenetic mediated host responses. The aim of present study was to explore the changes in epigenetic signatures of Caco-2 cells by probiotic strains (Lactobacillus rhamnosus MTCC 5897: LR, Lactobacillus fermentum MTCC 5898: LF and their mixture: RF), respectively, or during challenge with Escherichia coli (ATCC 14948) using exclusion, competition and displacement assays. Adenocarcinoma intestinal epithelial Caco-2 cells were treated with LR, LF, RF and $E$. coli for $6 \mathrm{~h}$, respectively. Caco-2 cells were also challenged with $E$. coli and probiotic lactobacilli during exclusion, competition and displacement assays. Finally, global epigenetic modifications by acetylation of $\mathrm{H} 4$ and $\mathrm{H} 3$ histone proteins and DNA methylation patterns were determined. Probiotic-treated Caco-2 cells displayed significant $(p<0.01)$ reduction in percent global $\mathrm{H} 4$ and $\mathrm{H} 3$ acetylation, respectively, in contrast to their elevated $(p<0.05)$ levels after $E$. coli infection. On the other hand, a remarkable $(p<0.01)$ decrease in percent $\mathrm{H} 4$ and $\mathrm{H} 3$ acetylation were observed when $E$. coli were excluded, competed or displaced by lactobacilli strains. No changes in the global DNA methylation patterns were observed in Caco-2 cells after exposure to probiotic strains or E. coli, respectively, but surprisingly, their levels increased significantly $(p<0.05)$ when lactobacilli-treated cells were challenged with $E$. coli during exclusion or competition than displacement assays. Probiotic L. rhamnosus and L. fermentum modulated the host epigenetic signatures via global histone acetylation individually or during $E$. coli challenge by exclusion, competition and displacement assays. Whilst on the other hand changes in global DNA methylation patterns were obtained significantly during probiotic treatment with $E$. coli in exclusion and competition protocols.
\end{abstract}

Keywords DNA methylation $\cdot$ Epigenome $\cdot$ Histone acetylation $\cdot$ Lactobacilli $\cdot$ Caco-2

\section{Introduction}

Epigenetics is the study of mitotically and meiotically heritable changes in gene function that are independent of DNA sequence (Bhat and Kapila 2017). The epigenome or the overall epigenetic state of an organism is just as important to normal development as is the contribution of the parent genome.

Electronic supplementary material The online version of this article (https://doi.org/10.1007/s13213-019-01451-0) contains supplementary material, which is available to authorized users.

Rajeev Kapila

rkapila69@rediffmail.com

1 Animal Biochemistry Division, ICAR-National Dairy Research Institute, Karnal, Haryana 132001, India
The relationship between individual genotype and the environment is very essential for the proper host functioning and any perturbation in this association may have lethal consequences (Feinberg 2008; Tollefsbol 2017). Epigenomic reprogramming of the host genome is involved in various life processes such as development, regeneration and postpartum life of higher eukaryotic organisms (Al Akeel 2013). Environmental factors like gut microbiota and their metabolites, nutrients, toxins, infections and hypoxia can have profound effects on the epigenetic signature which may trigger host susceptibility to different disorders (Safronova and Morita 2010; Vel Szic et al. 2015). Epigenetics is therefore considered to be at the epicentre of modern medicine as it helps in understanding the disease aetiology (Feinberg 2008; Tollefsbol 2017). Studies have shown that the bioactive components and gut microbes alter the epigenetic signatures of 
host cells through a variety of mechanisms which induced wide host responses ranging from inflammatory cascades to gut-brain axis modulation (Bhat and Kapila 2017; Zhou et al. 2017). The major epigenetic modifications that alter accessibility of DNA to transcriptional machinery and influence gene expression are acetylation, methylation, phosphorylation, biotinylation and RNA interference (Wang et al. 2018). These processes involve chromatin uncoiling and recoiling by the addition and removal of specific chemical groups which subsequently influence the host cells (Wong et al. 2017). Therefore, methylated DNA, acetylated proteins, micro RNA (miRNA), specific substrates, cofactors and enzymes involved in various biochemical reactions associated with these processes could serve as important biomarkers for detection of various diseases (Qureshi et al. 2010; Tollefsbol 2017).

Recently, it has been found that various infectious agents like Epstein-Barr virus, hepatitis viruses B and C, human papilloma virus, polyomaviruses, Streptococcus bovis, Chlamydia pneumoniae, Campylobacter rectus and Helicobacter pylori induce their lethal consequences through epigenetic changes (Oka et al. 2011). Similarly, the metabolic imbalances and inflammatory cascades have been linked to aberrant epigenetic patterns in the host cells (Safronova and Morita 2010). Even imbalanced gut microbiome (dysbiosis) set in aberrant epigenetic changes in the host cells that are linked with progression of many diseases such as systemic immune diseases including IBD, multiple sclerosis (MS) and autoimmune diseases (Rogers et al. 2016; Rooks and Garrett 2016; Lee et al. 2017b). The positive manipulation or restoration of altered gut microbiome through transplantation of the healthy gut microbiome or probiotics is therefore considered the best remedy to overcome such health problems and could constitute an effective therapeutic strategy. Probiotic and their metabolites in particular have been recently under tremendous consideration as these form important part of host gut environment and thus may have massive influence on host epigenome. However, the mechanistic understanding of such modifications is still in infancy and thus lot need to be done to comprehend such changes.

The present study was therefore conducted to understand the epigenetic mechanism/modification viz. global $\mathrm{H} 3$ and $\mathrm{H} 4$ acetylation and DNA methylation induced by two indigenous bacterial probiotic strains Lactobacillus rhamnosus (LR: MTCC 5897) and Lactobacillus fermentum (LF: MTCC 5898). Both the lactobacilli strains has been previously characterised for various probiotic attributes and have shown different efficacies (Sharma et al. 2014a; Sharma et al. 2014b; Saliganti et al. 2015). L. rhamnosus was reported to alleviate the ovalbumin induced allergy in newborn mice at suckling weaning transition and enhance immune functions with reduced oxidative stress in ageing mice as well resistance to E. coli infection (Sharma et al. 2014a; Saliganti et al. 2015).
On the other hand L. fermentum in the form of fermented milk showed potential to boost immunity, improve antioxidant capacity and resistance against pathogenic infection in aged murine model (Sharma et al. 2014b). Besides, both the probiotic strains showed ability to prevent the $E$. coli-induced aberration in the intestinal barrier integrity under in vitro conditions (in press). Therefore, it was necessary at the moment to investigate how the probiotic lactobacilli strains interferes with the host $\mathrm{H} 3$ and $\mathrm{H} 4$ acetylation and DNA methylation patterns when incubated individually with host intestinal epithelial cells or during exclusion, competition and displacement assays in presence of $E$. coli as an inflammatory agent.

\section{Material and methods}

\section{Epithelial cell culture}

The colonic adenocarcinoma enterocytes like Caco- 2 cells were procured from the National Centre for Cell Science (NCCS, Pune, India) and were maintained in Dulbecco's modified Eagle's medium (DMEM) supplemented with $10 \%$ fetal bovine serum along with $1 \%$ antibiotic solution (containing $1 \%$ streptomycin and penicillin $\mathrm{G}$ each and amphotericin $0.03 \%$ ) and incubated at $37{ }^{\circ} \mathrm{C}$ in $10 \% \mathrm{CO}_{2}$ humidified atmosphere. The confluent cells with 20-30 passage numbers were used for this study.

\section{Bacterial strains and their propagation}

The probiotic L. rhamnosus and L. fermentum used in the present study were previously isolated from house hold curd and faeces of a 10-month-old infant, respectively. Both bacteria were grown overnight at $37{ }^{\circ} \mathrm{C}$ in Mann-Rogosa-Sharpe (MRS) broth (Himedia Laboratories, Mumbai India), centrifuged at $3000 \mathrm{rpm}$ to pellet down the bacteria and washed thrice with saline. Final density of bacteria was set at $10^{9} \mathrm{CFU} / \mathrm{mL}$ in antibiotic-free DMEM for experiments.

On the other hand, E. coli (ATCC 14948) used to induce the inflammation was obtained from National Collection of Dairy Cultures (NCDC; NDRI, Karnal Haryana) and was grown in nutrient broth overnight at $37{ }^{\circ} \mathrm{C}$ on the day of experiment. The final count of $E$. coli bacteria was set also at $10^{9} \mathrm{CFU} / \mathrm{mL}$ in antibiotic-free DMEM for the experimental purpose.

\section{Histone acetylation assay}

The effect of probiotic lactobacilli on the histone acetylation of intestinal cells was determined by assessing the global histone $4(\mathrm{H} 4)$ and histone $3(\mathrm{H} 3)$ acetylation levels. To conduct the acetylation study, probiotic cultures $L$. rhamnosus and $L$. fermentum were grown overnight in MRS broth, whereas 
E. coli was propagated in nutrient broth. On the day of experiment, live probiotic strains L. rhamnosus (LR: $1 \times 10^{9} \mathrm{CFU} /$ $\mathrm{ml}$ ), L. fermentum (LF: $\left.1 \times 10^{9} \mathrm{CFU} / \mathrm{ml}\right)$ and their mixture (RF: LR: $1 \times 10^{9} \mathrm{CFU} / \mathrm{ml}+\mathrm{LF}: 1 \times 10^{9} \mathrm{CFU} / \mathrm{ml}$ ) as well as E. coli $\left(1 \times 10^{9} \mathrm{CFU} / \mathrm{ml}\right)$ were suspended in antibiotic-free DMEM and incubated separately with confluent Caco- 2 cells for $6 \mathrm{~h}$. After incubation, cells were rinsed thrice with PBS $(\mathrm{pH} 7.4)$ and were processed for the assessment of global changes in histone $\mathrm{H} 4$ and $\mathrm{H} 3$ acetylation levels using EpiQuik global histone $\mathrm{H} 3$ and $\mathrm{H} 4$ acetylation assay kits. These epigenetic changes were also determined in challenge study when $E$. coli were excluded, competed or displaced with lactobacilli. In exclusion mode of treatment, Caco-2 cells were pre-treated with respective probiotic lactobacilli strains or their mixture for $6 \mathrm{~h}$ followed by removal of lactobacilli containing media and then exposing to $E$. coli for same duration. In competition assay, Caco-2 cells were simultaneously treated with either of probiotic strains individually or their mixture with $E$. coli for $6 \mathrm{~h}$. Whereas in displacement assay, intestinal cells were pre-exposed to $E$. coli for $6 \mathrm{~h}$, and then after aspirating the media, cells were incubated further for $6 \mathrm{~h}$ with probiotic L. rhamnosus, L. fermentum individually or their mixture, respectively. The 6-h duration of exposure of probiotic lactobacilli and $E$. coli with $10^{9} \mathrm{CFU} / \mathrm{ml}$ dose was selected on the basis of optimal expression of tight junctional genes associated with barrier functions during temporal exposure of probiotic strains or E. coli to Caco-2 cells (Data in supplementary file).

\section{Histone extraction and quantification of $\mathrm{H} 4$ and $\mathrm{H3}$ acetylation levels}

The intestinal epithelial Caco-2 cells exposed to either probiotic lactobacilli or E. coli and untreated cells were lysed and subjected to $\mathrm{H} 3$ and $\mathrm{H} 4$ histone quantification by using EpiQuik global histone $\mathrm{H} 3 / \mathrm{H} 4$ acetylation assay kits (Epigentek Group, Farmingdale, NY, USA), respectively, according to the manufacturer's instructions. Briefly, the treated or untreated cells were initially rinsed thrice with PBS $(\mathrm{pH}$ 7.4) and detached from the adherent surface by gentle pipetting. The cells were homogenised with diluted lysis buffer (200 $\mu \mathrm{l} / 10^{6}$ cells) and incubated on ice for $5 \mathrm{~min}$. Cell debris containing nuclei were obtained by centrifugation $(12,000 \mathrm{rpm})$ followed by addition of lysis buffer $(20 \mu \mathrm{l})$ and extraction/glycerol $(60 \mu \mathrm{l})$ solution. After 5-min incubation, supernatant was collected by centrifugation and added with $100 \%$ TCA solution (1:4) to precipitate the protein. The protein pellet obtained was washed with acetone twice and then air dried before dissolving it in distilled water $(20 \mu \mathrm{l})$.

The changes in the $\mathrm{H} 3$ and $\mathrm{H} 4$ levels were estimated in histone extracts using the manufacturer's instructions. Briefly, the protein concentration in each control or treated sample was estimated by Bradford's method and was adjusted to $200 \mathrm{ng} / \mu \mathrm{l}$. Equal volume $(5 \mu \mathrm{l})$ of protein solution was spread in the central area of each well of 96-well plate (Epigentek, USA) with blank wells having histone buffer $(5 \mu \mathrm{l})$. For the positive controls, standard acetylated histone $\mathrm{H} 3$ and $\mathrm{H} 4(60 \mu \mathrm{g} / \mathrm{ml})$ after dilution to $20-120 \mathrm{ng} / \mu \mathrm{l}$ were added. The plate was incubated at $37{ }^{\circ} \mathrm{C}$ for $60-90 \mathrm{~min}$ for drying and later $150 \mu \mathrm{l}$ of blocking buffer was added. To each well, $50 \mu$ of diluted capture antibody (1:100) was added and was incubated (1 h, RT) on an orbital shaker (75 rpm). After proper washing, $50 \mu$ l of diluted HRP-conjugated detection antibody (1:1000 diluted) was added to each well and the plate was kept for 30-min incubation at room temperature. The wells were again rinsed with wash buffer and $100 \mu$ l of developing solution was added. Finally, reaction was blocked by adding stop solution $(50 \mu \mathrm{l} /$ well $)$ and plates were read at $450 \mathrm{~nm}$ (Epoch, BioTek, USA). The results were expressed as relative $\mathrm{H} 3$ and $\mathrm{H} 4$ acetylation percentage between treated and untreated control epithelial cells.

\section{DNA methylation assay}

The change in the DNA methylation patterns of human intestinal cells upon exposure to probiotic lactobacilli and E. coli as described above was determined by measuring the global DNA methylation status of treated and untreated cells according to manufacturer's instructions. Briefly, treated and untreated Caco-2 cells were washed thrice in PBS (pH 7.4) and cellular lysate was obtained by addition of $10 \% \operatorname{SDS}(50 \mu \mathrm{l})$ for $30 \mathrm{~min}$ at room temperature. To the cell lysate, $5 \mathrm{M} \mathrm{NaCl}$ $(200 \mu \mathrm{l})$ and of phenol/chloroform/isoamyl alcohol solution $(25: 24: 1,100 \mu \mathrm{l})$ was added for phase separation and top aqueous phase containing DNA was collected. To pellet down DNA, aqueous phase was added with ice cold iso-propanol $(500 \mu \mathrm{l})$ and mixed gently followed by incubation at $-20^{\circ} \mathrm{C}$ for $30 \mathrm{~min}$. The DNA pellet was obtained by centrifugation and washed, air dried and dissolved in nuclease-free water $(20 \mu \mathrm{l})$. To remove any traces of RNA contamination, RNase I ( $1 \mu \mathrm{l}, 10 \mathrm{U} / \mu \mathrm{l})$ was added to DNA solution and was then processed immediately or stored at $-20{ }^{\circ} \mathrm{C}$ for later use.

The change in global DNA methylation was estimated by Methylamp global DNA methylation quantification ultra kit (Epigentek Group Inc., USA) following the manufacturer's instructions. Briefly, the DNA concentration in each control or treated sample was adjusted to $80 \mathrm{ng} / \mu \mathrm{l}$. Each well was added with $28 \mu$ of DNA binding solution followed by addition of $2 \mu \mathrm{l}$ of DNA (160 ng/well). Similarly, positive control wells were added with $2 \mu$ l of standard diluted samples, whereas negative control wells added with same volume of negative control DNA. The plate was incubated at $37{ }^{\circ} \mathrm{C}$ for $40 \mathrm{~min}$ followed $30-40$-min incubation at $60^{\circ} \mathrm{C}$ to evaporate and dry the wells. To each dried well, blocking buffer $(150 \mu \mathrm{l})$ was added and incubated at $37^{\circ} \mathrm{C}$ for $30 \mathrm{~min}$. Each well was 
aspirated and rinsed thrice with $1 \times$ wash buffer followed by addition of capture antibody (1:1000 with 1× wash buffer). After primary antibody incubation ( $1 \mathrm{~h}, \mathrm{RT})$, each well was again washed and added with diluted HRP-conjugated detection antibody ( $50 \mu \mathrm{l}, 1: 5000)$ for $30 \mathrm{~min}$ at room temperature. After washings, each well was added with enhancer solution $(50 \mu \mathrm{l})$ for $30 \mathrm{~min}$ followed by developing solution $(100 \mu \mathrm{l})$ and the plate was incubated for 1-5 min away from light for colour development. Finally, horseradish peroxidise catalysed reaction was impeded with stop solution $(50 \mu \mathrm{l})$ and the plate was read at $450 \mathrm{~nm}$. The results were expressed as percent (\%) DNA methylation levels between treated and untreated Caco2 cells.

\section{Statistical analysis}

Data were analysed using the GraphPad Prism (version 5.01). The results were presented as means \pm SEM (standard errors of the mean). One-way analysis of variance (ANOVA) was used for statistical analysis using Tukey-Kramer post hoc test to know the statistical significance at $p<0.05$ for multiple comparison amongst means.

\section{Results}

\section{Global histone acetylation and DNA methylation of intestinal cells upon exposure to probiotic lactobacilli and $E$. coli}

Human intestinal Caco-2 cells were treated with probiotic L. rhamnosus $\left(1 \times 10^{9} \mathrm{CFU} / \mathrm{ml}\right)$, L. fermentum $(1 \times$ $10^{9} \mathrm{CFU} / \mathrm{ml}$ ) and their mixture (RF: L. rhamnosus $+L$. fermentum) and E. coli $\left(1 \times 10^{9} \mathrm{CFU} / \mathrm{ml}\right)$, respectively, for $6 \mathrm{~h}$ to assess their effect on the global histone acetylation.

Treatment of intestinal epithelial cells with LR LF and RF significantly $(p<0.01)$ reduced the relative $\mathrm{H} 4$ acetylation to $56.7 \pm 8.1,45.6 \pm 7.4$ and $57.9 \pm 11.3 \%$, respectively, compared to untreated control cells (Fig. 1a). Likewise, in case of H3 acetylation, treatment of LR, LF and RF to intestinal Caco-2 also depicted significant $(p<0.05)$ suppression by $56.4 \pm 2.3,67.0 \pm 10.4$ and $61.6 \pm 12.6 \%$, respectively, in the relative global acetylation level compared to untreated cells (Fig. 1b). On the contrary, both $\mathrm{H} 4$ and H3 acetylation enhanced significantly $(p<0.05)$ by $145.2 \pm 12.5$ (Fig. 2$)$ and $179.3 \pm 25.2 \%$ (Fig. 3), respectively, when confluent Caco-2 cells were exposed separately to live $E$. coli for $6 \mathrm{~h}$.

The treatment of human intestinal Caco- 2 cells with either of the probiotic L. rhamnosus, L. fermentum or their mixture for $6 \mathrm{~h}$ showed statistically insignificant changes in relative global DNA methylation percentages compared to untreated cells as represented in Fig. 1c. Similarly insignificant modifications were observed in DNA methylation status by E. coli exposure alone (Fig. 4).

\section{Impact of probiotic lactobacilli treatment on global histone acetylation during challenge with $E$. coli}

In exclusion assay, pre-treatment of intestinal Caco-2 cells with LR, LF and RF followed by $E$. coli significantly $(p<0.01)$ reduced the percent $\mathrm{H} 4$ acetylation levels to 28.5 $\pm 2.8,30.7 \pm 3.1$ and $44.7 \pm 5.1$, respectively (Fig. 2a), compared to $E$. coli-treated $(145.2 \pm 12.5)$ cells. Similarly, the simultaneous incubation of inflammatory agent $E$. coli with probiotic strains (LR, LF and RF) on Caco-2 cells during competition assay remarkably $(p<0.01)$ suppressed $(52.0 \pm$ $12.0,57.8 \pm 13.9$ and $42.9 \pm 7.8 \%$ ) the global $\mathrm{H} 4$ acetylation, respectively (Fig. 2b), compared to cells inflamed with $E$. coli alone $(145.18 \pm 12.5 \%)$. Likewise, displacement of $E$. coli by LR, LF and RF on intestinal epithelial Caco-2 cells during posttreatment with probiotic strains significantly $(p<0.01)$ diminished global acetylation of $\mathrm{H} 4$ histone proteins by $29.0 \pm 5.1,31.2 \pm 6.3$ and $66.2 \pm 5.0 \%$, respectively, compared to $145.2 \pm 12.5 \%$ acetylation with $E$. coli (Fig. 2c).

In case of global $\mathrm{H} 3$ acetylation, the epigenetic modifications have shown significant $(p<0.05)$ reduction of $71.0 \pm$ $27.1,64.0 \pm 18.5$ and $73.4 \pm 23.2 \%$, respectively (Fig. 3a), on pre-incubation with LR, LF and RF during exclusion as compared to $179.3 \pm 25.2 \%$ by $E$. coli alone. Similarly during competition assay, $\mathrm{H} 3$ acetylation levels in host Caco-2 cells also diminished significantly $(p<0.01)$ to $74.6 \pm 15.9,39.2 \pm$ 6.9 and $30.5 \pm 9.4 \%$, respectively (Fig. $3 b$ ). In the same manner, $\mathrm{H} 3$ acetylation patterns of Caco- 2 cells declined significantly $(p<0.05)$ to $80.3 \pm 9.5,53.2 \pm 8.0$ and $75.3 \pm 10.3 \%$ when displacement of $E$. coli was attempted with respective probiotic lactobacilli treatments (Fig. 3c).

\section{Global DNA methylation status of human intestinal cells challenged with $E$. coli}

Pre-exposure of Caco-2 cells to probiotic L. rhamnosus, L. fermentum and their combination significantly $(p<0.05)$ elevated the global DNA methylation to $131.9 \pm 11.5,145.2$ \pm 4.9 and $128.8 \pm 5.0 \%$, respectively, compared to $83.9 \pm$ $2.6 \%$ when cells were exposed to $E$. coli alone as an inflammatory agent (Fig. 4a). On other hand, concurrent treatment of LR and RF with $E$. coli considerably $(p<0.01)$ enhanced the DNA methylation by $205.6 \pm 27.8$ and $286.0 \pm 27.8 \%$, respectively, compared to E. coli control. However, co-treatment of LF with $E$. coli had insignificant effect on DNA methylation of intestinal Caco-2 cells compared to E. coli exposed cells or control cells (Fig. 4b). Interestingly, in case of displacement assay, none of probiotic strains or their combination was found to alter the global methylation epigenetic signatures of 


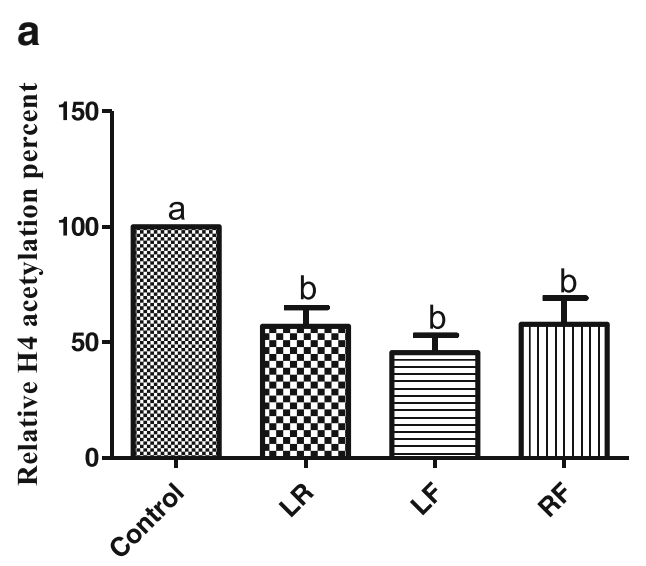

Treatments b

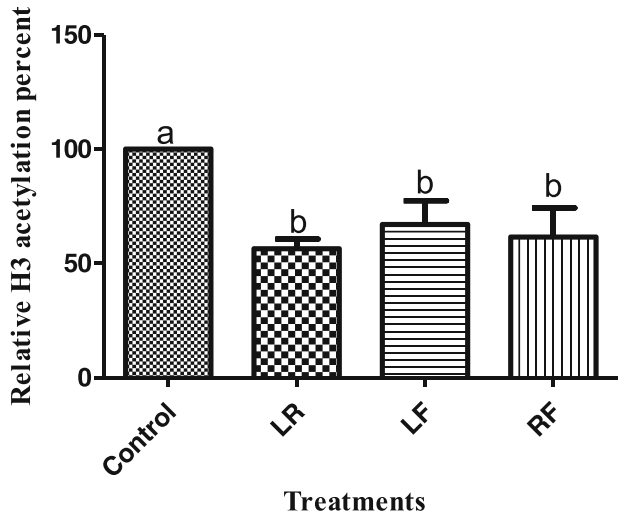

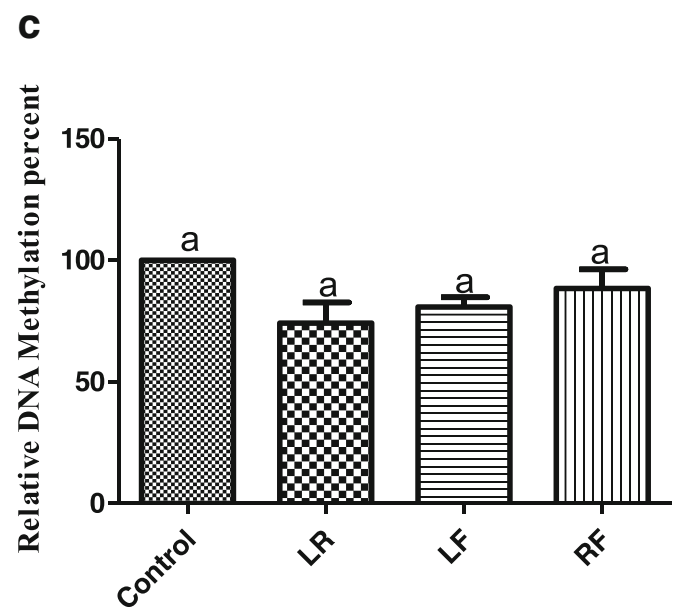

Treatments

Fig. 1 Effect of probiotic lactobacilli (L. rhamnosus: LR, L. fermentum: LF and their mixture: RF) on global epigenetic signatures of human intestinal epithelial cells. a $\mathrm{H} 4$ acetylation. b $\mathrm{H} 3$ acetylation. c DNA

host intestinal epithelial cells compared to $E$. coli-exposed Caco-2 cells (Fig. 4c).

\section{Discussion}

Epigenetics predominantly emphasise on the mechanisms that could mould the chromatin structures and regulate gene expression with stability by adapting cells to the environment without changing the nucleotide sequence (Canani et al. 2012). In a broader context, there is a mounting interest in probiotics to understand their involvement in epigenetic mechanisms so that more specific and efficacious therapeutic strategies can be developed in the prevention and treatment of different diseases. Epigenetic mechanisms integrate environmental changes at the cellular level, thereby enabling cellular plasticity (Flavahan et al. 2017). There are basically three distinct but intimately interacting epigenetic mechanisms that can change the expression profile of key genes essential for methylation. Values are expressed as mean $\pm \operatorname{SEM}(n=3$ for each treatment) and values with different superscript letters are significantly different at $p<0.01$

physiological and pathologic processes (McKay and Mathers 2011). Epigenetic modifications contribute significantly in the modulation of gene transcription through DNA methylation, histone modifications and non-coding microRNAs (miRNA). DNA methylation is generally associated with the suppression of gene transcription, whereas acetylation of histones is predominantly associated with gene induction (Woo et al. 2017). RNA interference on other hand is an epigenomic process where genes are turned off posttranscriptionally by a group of small, noncoding, endogenous miRNA (Bhat and Kapila 2017). Although the area of epigenetics has come under tremendous contemplation recently but still scanty literature is available about association of host epigenome with probiotics and is thus least understood.

Under present investigation, the impact of probiotic bacteria L. rhamnosus (LR; MTCC 5897) and L. fermentum (LF; MTCC 5898) on the host epigenome viz. global H3 and H4 acetylation and DNA methylation were assessed in the presence of inflammatory stimulus of $E$. coli. Human colon 
a

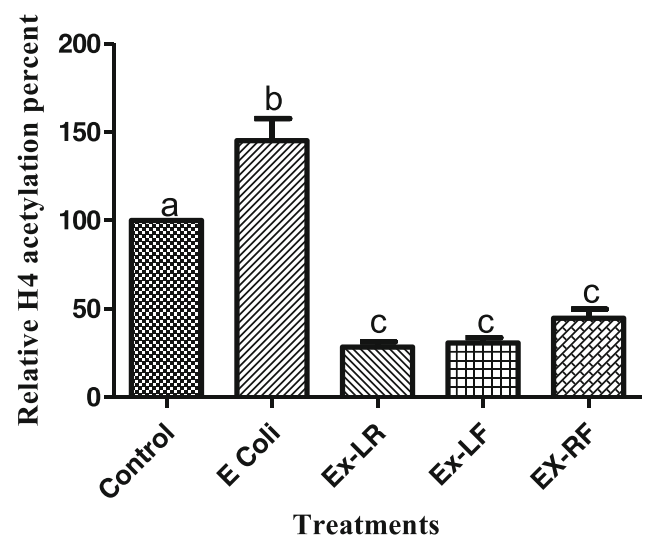

b

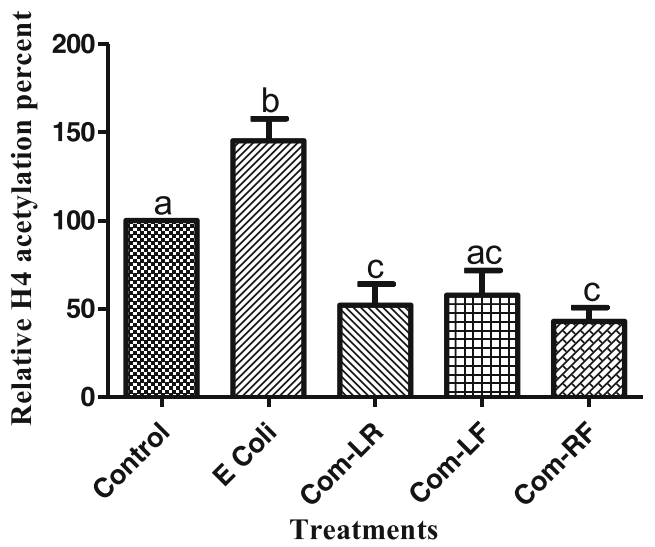

C

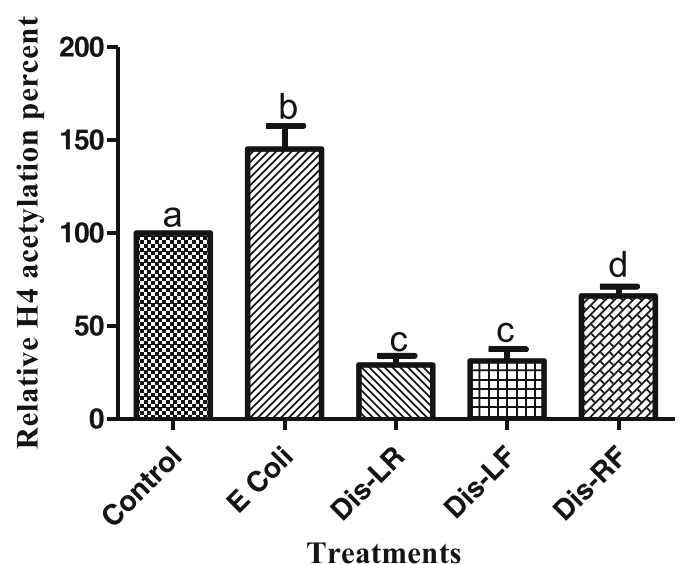

Fig. 2 Effect of probiotic lactobacilli (L. rhamnosus: LR, L. fermentum: LF and their mixture: RF) during challenge with E. coli on global H4 histone acetylation in intestinal Cao-2 cells. a Exclusion (Ex). b
Competition (Com). c Displacement (Dis). Values are expressed as mean $\pm \operatorname{SEM}(n=3$ for each treatment) and values with different superscript letters are significantly different at $p<0.01$ epithelial Caco-2 cells treated with probiotic strains LR, LF and their mixture RF individually for $6 \mathrm{~h}$ displayed significant reduction in the percent global histone $\mathrm{H} 4$ and $\mathrm{H} 3$ acetylation in contrast to remarkably high acetylation observed during E. coli exposure. The increased $\mathrm{H} 3$ and $\mathrm{H} 4$ acetylation of histone proteins by E. coli exposure might suggest the enhanced inflammatory response by host cells against inflammatory conditions in contrast to suppressed levels of $\mathrm{H} 3$ and $\mathrm{H} 4$ acetylation patterns indicating the healthy environment set in by probiotic lactobacilli strains. In context to this interpretation, we have earlier established that probiotic strains $L$. rhamnosus and L. fermentum were able to alleviate the host inflammation by suppressing the expression of inflammatory interleukin-4 and interleukin-10 in contrast to increased interferon- $\gamma$ levels that might have been modulated by involving epigenetic machinery (Sharma et al. 2014a; Sharma et al. 2014b; Saliganti et al. 2015). Previously, Lee et al. (2017a) reported that the commensal probiotic lactobacilli strains Lactobacillus gasseri and Lactobacillus reuteri in a strain- specific manner modulated the expression of DEFB1 (Defensin Beta 1) gene by changing the acetylation patterns at specific regions in vaginal VK2/E6E7 epithelial cells. On one hand, L. gasseri showed enhanced expression of DEFB1 due to enrichment of active histone marks such as $\mathrm{AcH} 3$ and H2A.Z whilst on the other treatment with $L$. reuteri decreased such active histone marks that subsequently resulted in reduced DEFB1 expression. Increased global $\mathrm{H} 3$ and $\mathrm{H} 4$ acetylation levels were found in human macrophage cells upon exposure to inflammatory agents such as ethanol and LPS that elevated the pro-inflammatory cytokine profile of host cells leading to chronic inflammation (Kendrick et al. 2010). Enhanced H4 acetylation were also seen when gastric epithelial cells were exposed to $H$. pylori emphasising that chromatin alterations might contribute to the pathogenic mechanisms underlying the development and progression of $H$. pylori-associated diseases (Xia et al. 2008). In similar context, Ghadimi et al. (2012) observed significant reduction in the global H3 acetylation levels when HT-29/B6 or T84 monolayers in 3D 

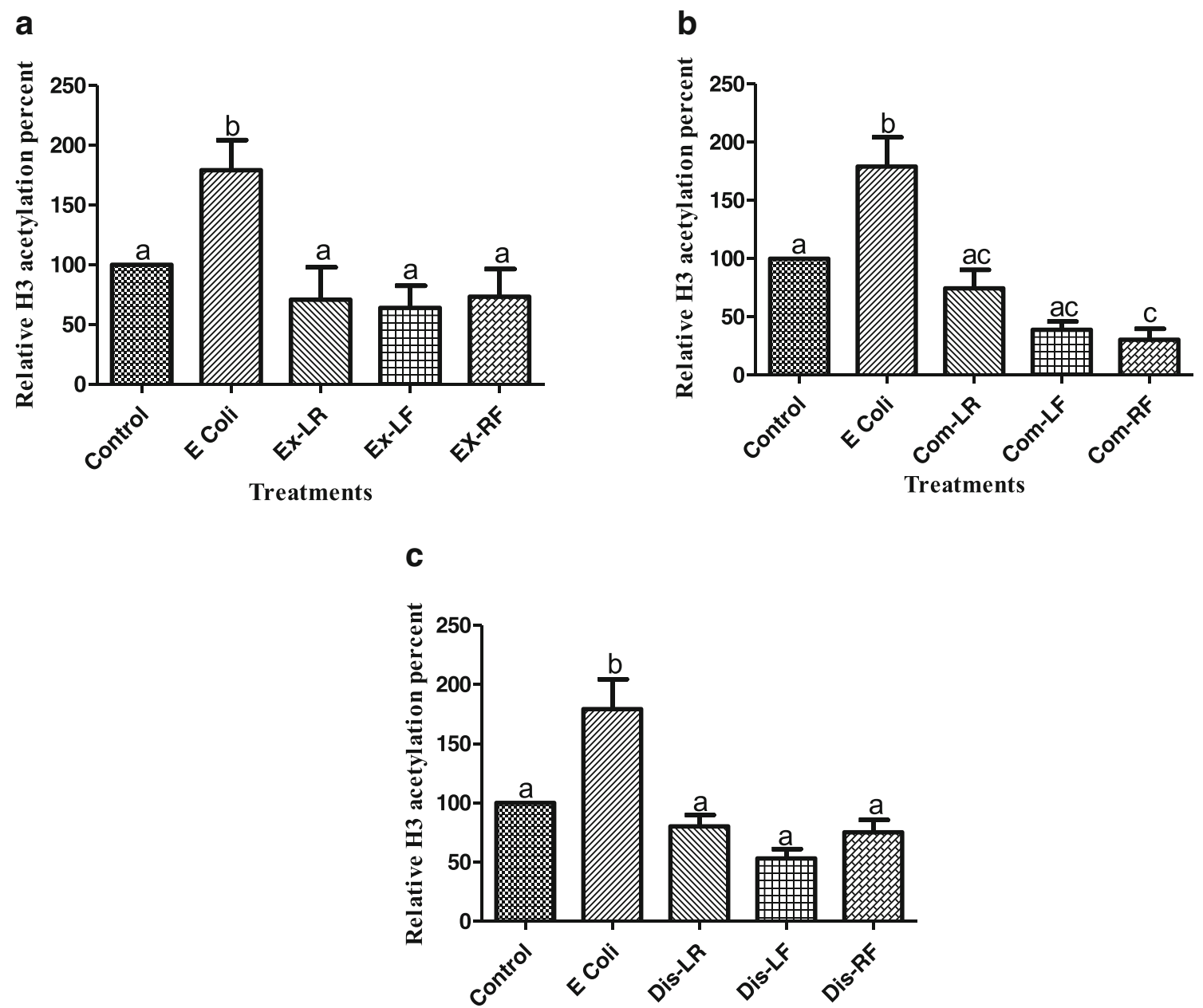

Fig. 3 Effect of probiotic lactobacilli (L. rhamnosus: LR, L. fermentum: $\mathrm{LF}$ and their mixture: RF) during challenge with $E$. coli on global $\mathrm{H} 3$ histone acetylation in intestinal Cao-2 cells. a Exclusion (Ex). b
Treatments

Competition (Com). c Displacement (Dis). Values are expressed as mean $\pm \operatorname{SEM}$ ( $n=3$ for each treatment) and values with different superscript letters are significantly different at $p<0.05$ culture systems were separately treated with probiotic $B$. breve or L. rhamnosus, respectively. The decreased acetylation levels were responsible for alleviating the inflammatory cascade set in inflammatory stimulus that subsequently downregulate the expression of the inflammatory bowel disease causing factors. Recent evidences have further consolidated the perception that commensal gut microflora has a critical role in gut homeostasis and alteration in their composition is majorly associated with the development of gut diseases through host cell epigenome modulation. Depletion of gut microbiota by broad-spectrum antibiotic treatment induced massive change in the global epigenetic signatures of small intestinal cells including the several histone modifications at specific enhancer and promoter sites (Gury-BenAri et al. 2016). In earlier study, the interdependence of commensal bacteria and histone deacetylases in the regulating the intestinal homeostasis was also demonstrated, which clearly suggested that histone acetylation of host genes might change in the presence of commensal gut microbes (Alenghat and Artis 2014). Microbe- derived metabolites such as acetate and butyrate when supplemented under in vitro and in vivo conditions also have been established to modulate host epigenome by changing the acetylation patterns of cells (Bhat and Kapila 2017). The gut microbes and their fermentation products were very essential in the attenuation of colorectal tumorigenesis by changing the histone acetylation patterns and the expression of associated genes (Donohoe et al. 2012; Donohoe et al. 2014). Spermine secretion by gut microbes $B$. thetaiotaomicron and Fusobacterium varium have been found to modulate the histone acetylation, which subsequently showed beneficial effects on ageing and age-related memory deficits (Noack et al. 2000; Stilling et al. 2014). However, in contrast to present findings, Bourassa et al. (2016) demonstrated the increased acetylation patterns induced by butyrate derived from Clostridium, Eubacterium and Butyrivibro that subsequently improved the memory impairment and learning power by increasing the expression of learning-associated genes in Alzheimer mice model. However, Rada-Iglesias et al. (2007) 

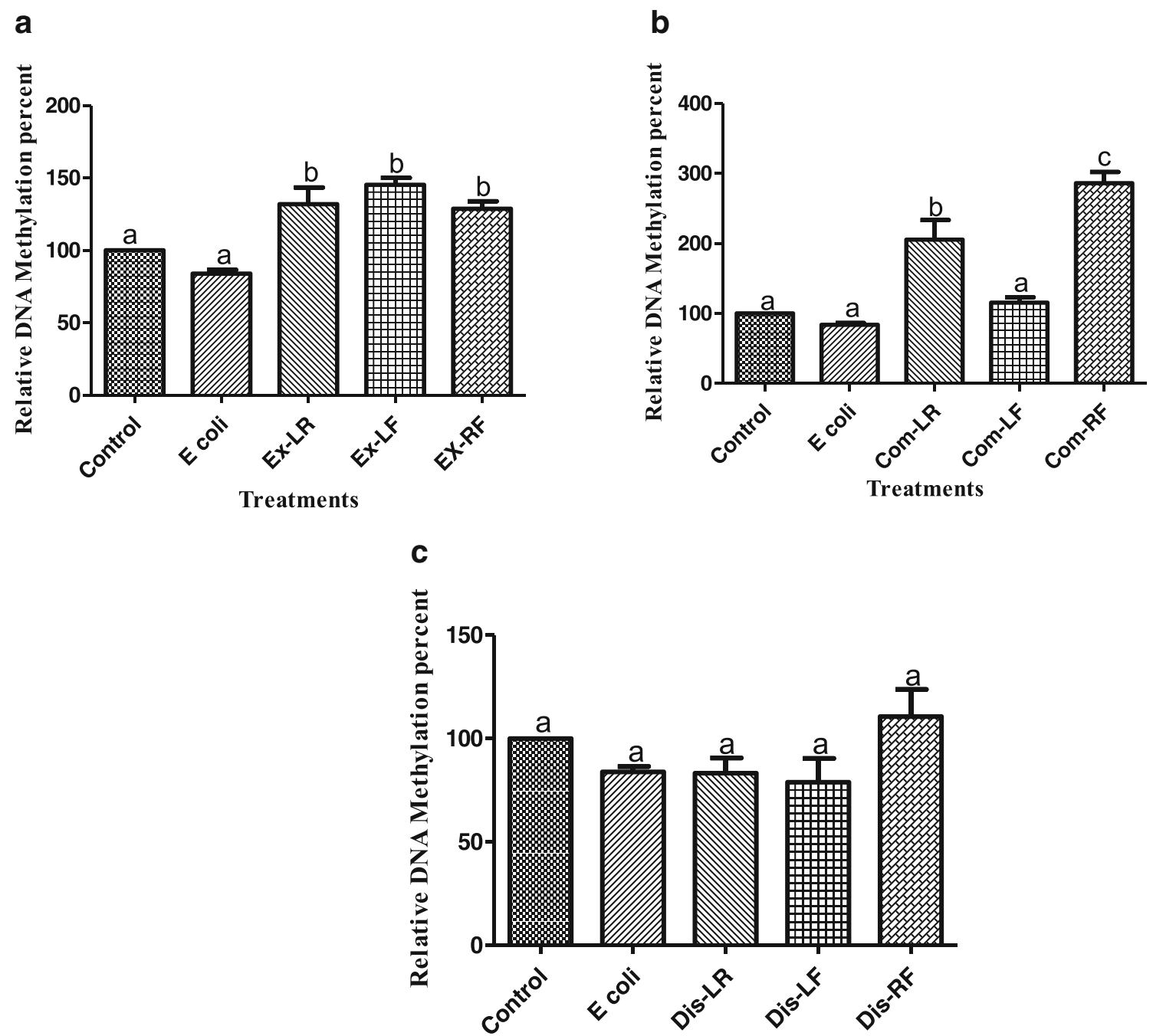

Treatments

Fig. 4 Global DNA methylation patterns in intestinal epithelial cells with probiotic lactobacilli (L. rhamnosus: LR, L. fermentum: LF and their mixture: RF) during challenge with E. coli. a Exclusion (Ex). b
Competition (Com). c Displacement (Dis). Values are expressed as mean $\pm \operatorname{SEM}(n=3$ for each treatment) and values with different superscript letters are significantly different at $p<0.05$ highlighted the role of butyrate derived from large bowel microbial fermentation in decreasing the histone acetylation at selected chromatin regions of hepatocarcinoma HepG2 and colon adenocarcinoma HT-29 cells.

Under present investigation, global DNA methylation which typically suppresses the gene activity appeared unaltered when intestinal epithelial Caco-2 cells were exposed to either of the probiotic strains or their mixture and live E. coli which is in accordance with previous finding where insignificant changes in global DNA methylation patterns were noticed upon exposure to commensal probiotics $B$. breve or L. rhamnosus under in vitro conditions (Ghadimi et al. 2012). Interestingly, they also reported insignificant changes in DNA methylation patterns of epithelial cells upon exposure to inflammatory agent LPS. However contrarily to present finding, Schmeck et al. (2005) reported the induction of DNA methylation changes in $H$. pylori-infected human endothelial cells that were involved in the regulation of host inflammatory response. Probiotic bacteria Lactobacillus acidophilus were also reported to change DNA methylome of intestinal cell which subsequently elevated the expression tumour-suppressing genes such as Icam 5, Clstn2, Ppm1e, Runx3, Timp3, Rgl1 and Rassfla and thereby avert colorectal cancer (Lightfoot et al. 2013). Differential DNA modification patterns were seen in human foetal and adult intestinal epithelial cell lines (H4 and NCM460, respectively) upon interaction with probiotics $L$. acidophilus and Bifidobacterium infantis or pathogenic bacteria Klebsiella spp. (Cortese et al. 2016). In a dietary intervention study with postmenopausal women, altering plasma levels of microbial metabolites folate were found to directly influenced lymphocyte DNA methylation levels (Jacob et al. 1998). Commensal gut microbes have very essential role in maintaining the DNA methylation patterns of important host genes such as TLR4 that are involved in 
sensing the external environmental stimuli that subsequently modulate host immune response (Takahashi et al. 2011). By modulating the DNA methylation patterns of host cells at TLR4 locus, the human intestinal cells were found to retaliate very poorly to external LPS stimulus reflecting the critical role of epigenome in countering the host responses.

Under the current investigation, epigenetic signatures of intestinal epithelial cells changed considerably when probiotic lactobacilli treated cells were challenged with $E$. coli. The global $\mathrm{H} 4$ and $\mathrm{H} 3$ histone acetylation levels were suppressed dramatically when human intestinal Caco-2 cells were treated with LR, LF and their mixture RF during challenge with E. coli under exclusion, competition and displacement milieu compared to E. coli exposure alone. This could be due to reduced inflammation of epithelial Caco-2 cells as probiotic strains may have inhibited $E$. coli from inducing its inflammatory signalling cascades. Similar to our observation, coincubation of transforming growth factor- $\beta 1$ (TGF- $\beta 1$ ) as an antiinflammatory agent with either non-pathogenic commensal enteric bacterial species $B$. vulgates or LPS on intestinal epithelial CMT93 cells reduced histone 3 hyper-acetylation which subsequently inhibited the expression of proinflammatory $I L-6$ gene (Haller et al. 2003). In the same way, Ghadimi et al. 2012 delineated a reversal in the LPS induced hyper-acetylation when commensal probiotic $B$. breve or L. rhamnosus were concurrently added with $3 \mathrm{D}$ co-culturing system of HT-29/B6 or T84 with PBMC cells.

In case of global DNA methylation patterns, lactobacilli strains were also able to enhance the host cell nuclear methylation levels when human intestinal colon cells (Caco-2) were treated with probiotic (LR, LF and RF) during exclusion and competition mode of treatment. However, no such prominent changes were observed when $E$. coli was incubated with epithelial cells prior (displacement assay) to probiotic lactobacilli treatment. This indicated maintenance of DNA methylation patterns in presence of inflammatory conditions causing genome silencing parallel to control group which could not even be reversed by posttreatment with probiotic strains. However, increased global DNA methylation during exclusion and competition assays depicted presence of healthier conditions due to prophylactic effect of probiotic with less expression of inflammatory genes. These results are in consonance with previous studies where the global methylation status increased considerably in kidney tissue of C57BL/6 $(\mathrm{H}-2 \mathrm{Ab})$ mice when administrated intraperitoneally with commensal bacteria Bifidobacterium adolescentis or B. longum before and during induction of ischemia and reperfusion injury (IRI), respectively (Andrade-Oliveira et al. 2015). Increased global DNA methylation levels were also observed when LPS and B. breve or L. rhamnosus were simultaneously added to HT-29/B6 and T84 cell monolayers (Ghadimi et al. 2012). Recently, increased methylation patterns of nitric oxide synthase (NOS) gene in Wistar rat burn injury model were reported when provided with combined therapy of glutamine and probiotics compared to control animals and thus protected them against burn-induced oxidative stress and inflammation (Gong et al. 2017). These epigenetic modifications in response to probiotic bacteria or in presence of inflammatory agent could directly or indirectly activate or inhibit the critical components of epigenomic machinery by interfering in the activity of enzymes such as methyltransferases, deacetylases, acetyltransferases, nucleases and serine-threonine protein kinases, which are central in reprogramming and/or in maintaining the posttranslation modification of histone and other proteins.

In conclusion, it has been established that exposure of intestinal epithelial Caco-2 cells to probiotic bacteria L. rhamnosus and L. fermentum brought out considerable changes in the core epigenetic signatures viz. histone acetylation and DNA methylation of host cells during challenge with inflammatory agent $E$. coli that might be responsible for the efficacy of these probiotic strains as established previously during alleviation of allergic response in weanlings and in combating immunosenescence in aged mice. However, lot need to ponder upon the gene specific modifications and the epigenetic machinery that could be involved in bringing out such modifications.

Funding We are grateful to Department of BioTechnology, Ministry of Science and Technology, New Delhi, for providing the necessary funds (BT/PR15109/PFN/20/1174/2015).

\section{Compliance with ethical standards}

Conflicts of interest The authors declare that they have no conflict of interest.

\section{Research involving human participants and/or animals N/A.}

Informed consent N/A.

Publisher's note Springer Nature remains neutral with regard to jurisdictional claims in published maps and institutional affiliations.

\section{References}

Al Akeel R (2013) Role of epigenetic reprogramming of host genes in bacterial pathogenesis. Saudi J Biol Sci 20:305-309

Alenghat T, Artis D (2014) Epigenomic regulation of host-microbiota interactions. Trends Immunol 35:518-525

Andrade-Oliveira V, Amanom MT, Correa-Costa M, Castoldi A, Felizardo RJ, de Almeida DC, Bassi EJ, Moraes-Vieira PM, Hiyane MI, Rodas AC, Peron JP (2015) Gut bacteria products prevent AKI induced by ischemia-reperfusion. J Am Soc Nephrol 26: $1877-1888$

Bhat MI, Kapila R (2017) Dietary metabolites derived from gut microbiota: critical modulators of epigenetic changes in mammals. Nutr Rev 75:374-389 
Bourassa MW, Alim I, Bultman SJ, Ratan RR (2016) Butyrate, neuroepigenetics and the gut microbiome: can a high fiber diet improve brain health? Neurosci Lett 625:56-63

Canani RB, Di Costanzo M, Leone L (2012) The epigenetic effects of butyrate: potential therapeutic implications for clinical practice. Clin Epigenetics 4:4

Cortese R, Lu L, Yu Y, Ruden D, Claud EC (2016) Epigenomemicrobiome crosstalk: a potential new paradigm influencing neonatal susceptibility to disease. Epigenetics 11:205-215

Donohoe DR, Collins LB, Wali A, Bigler R, Sun W, Bultman SJ (2012) The Warburg effect dictates the mechanism of butyrate-mediated histone acetylation and cell proliferation. Mol Cell 48:612-626

Donohoe DR, Holley D, Collins LB, Montgomery SA, Whitmore AC, Hillhouse A, Curry KP, Renner SW, Greenwalt A, Ryan EP, Godfrey VA (2014) Gnotobiotic mouse model demonstrates that dietary fiber protects against colorectal tumorigenesis in a microbiota-and butyrate-dependent manner. Cancer Discov 4: $1387-1397$

Feinberg AP (2008) Epigenetics at the epicenter of modern medicine. Jama 299:1345-1350

Flavahan WA, Gaskell E, Bernstein BE (2017) Epigenetic plasticity and the hallmarks of cancer. Science 357:aal2380

Ghadimi D, Helwig U, Schrezenmeir J, Heller KJ, Vrese M (2012) Epigenetic imprinting by commensal probiotics inhibits the IL-23/ IL-17 axis in an in vitro model of the intestinal mucosal immune system. J Leukoc Biol 92:895-911

Gong ZY, Yuan ZQ, Dong ZW, Peng YZ (2017) Glutamine with probiotics attenuates intestinal inflammation and oxidative stress in a rat burn injury model through altered iNOS gene aberrant methylation. Am J Transl Res 9:2535

Gury-BenAri M, Thaiss CA, Serafini N, Winter DR, Giladi A, LaraAstiaso D, Levy M, Salame TM, Weiner A, David E, Shapiro H (2016) The spectrum and regulatory landscape of intestinal innate lymphoid cells are shaped by the microbiome. Cell 166: 1231-1246

Haller D, Holt L, Kim SC, Schwabe RF, Sartor RB, Jobin C (2003) Transforming growth factor- $\beta 1$ inhibits non-pathogenic gram negative bacteria-induced NF- $\mathrm{kB}$ recruitment to the interleukin- 6 gene promoter in intestinal epithelial cells through modulation of histone acetylation. J Biol Chem 278:23851-23860

Jacob RA, Gretz DM, Taylor PC, James SJ, Pogribny IP, Miller BJ, Henning SM, Swendseid ME (1998) Moderate folate depletion increases plasma homocysteine and decreases lymphocyte DNA methylation in postmenopausal women. J Nutr 128:1204-1212

Kendrick SF, O'boyle G, Mann J, Zeybel M, Palmer J, Jones DE, Day CP (2010) Acetate, the key modulator of inflammatory responses in acute alcoholic hepatitis. Hepatology 51:1988-1997

Lee J, Jang A, Kim JW, Han JH, Chun BH, Jung HS, Jeon CO, Myung SC (2017a) Distinct histone modifications modulate DEFB1 expression in human vaginal keratinocytes in response to Lactobacillus spp. Probiotics Antimicrob Proteins 9:406-414

Lee ES, Song EJ, Nam YD (2017b) Dysbiosis of gut microbiome and its impact on epigenetic regulation. J Clin Epigenet 3(S1):14. https:// doi.org/10.21767/2472-1158.100048

Lightfoot YL, Yang T, Sahay B, Mohamadzadeh M (2013) Targeting aberrant colon cancer-specific DNA methylation with lipoteichoic acid-deficient Lactobacillus acidophilus. Gut Microbes 4:84-88

McKay JA, Mathers JC (2011) Diet induced epigenetic changes and their implications for health. Acta Physiol 202:103-118

Noack J, Dongowski G, Hartmann L, Blaut M (2000) The human gut bacteria Bacteroides thetaiotaomicron and Fusobacterium varium produce putrescine and spermidine in cecum of pectin-fed gnotobiotic rats. J Nutr 130:1225-1231

Oka T, Sato H, Ouchida M, Utsunomiya A, Yoshino T (2011) Cumulative epigenetic abnormalities in host genes with viral and microbial infection during initiation and progression of malignant lymphoma/leukemia. Cancers 3:568-581

Qureshi SA, Bashir MU, Yaqinuddin A (2010) Utility of DNA methylation markers for diagnosing cancer. Int J Surg 8:194-198

Rada-Iglesias A, Enroth S, Ameur A, Koch CM, Clelland GK, RespuelaAlonso P, Wilcox S, Dovey OM, Ellis PD, Langford CF, Dunham I (2007) Butyrate mediates decrease of histone acetylation centered on transcription start sites and down-regulation of associated genes. Genome Res 17:708-719

Rogers GB, Keating DJ, Young RL, Wong ML, Licinio J (2016) Wesselingh, S. From gut dysbiosis to altered brain function and mental illness: mechanisms and pathways. Mol Psychiatry 21:738

Rooks MG, Garrett WS (2016) Gut microbiota, metabolites and host immunity. Nat Rev Immunol 16:341

Safronova O, Morita I (2010) Transcriptome remodeling in hypoxic inflammation. J Dent Res 89(5):430-444

Saliganti V, Kapila R, Sharma R, Kapila S (2015) Feeding probiotic Lactobacillus rhamnosus (MTCC 5897) fermented milk to suckling mothers alleviates ovalbumin-induced allergic sensitisation in mice offspring. Br J Nutr 114:1168-1179

Schmeck B, Beermann W, van Laak V, Zahlten J, Opitz B, Witzenrath M, Hocke AC, Chakraborty T, Kracht M, Rosseau S, Suttorp N (2005) Intracellular bacteria differentially regulated endothelial cytokine release by MAPK-dependent histone modification. J Immunol 175:2843-2850

Sharma R, Kapila R, Dass G, Kapila S (2014a) Improvement in Th1/Th2 immune homeostasis, antioxidative status and resistance to pathogenic $E$. coli on consumption of probiotic Lactobacillus rhamnosus fermented milk in aging mice. Age 36:9686

Sharma R, Kapila R, Kapasiya M, Saliganti V, Dass G, Kapila S (2014b) Dietary supplementation of milk fermented with probiotic Lactobacillus fermentum enhances systemic immune response and antioxidant capacity in aging mice. Nutr Res 34: 968-981

Stilling RM, Dinan TG, Cryan JF (2014) Microbial genes, brain \& behaviour-epigenetic regulation of the gut-brain axis. Genes Brain Behav 13:69-86

Takahashi K, Sugi Y, Nakano K, Tsuda M, Kurihara K, Hosono A, Kaminogawa S (2011) Epigenetic control of host gene by commensal bacteria in large intestinal epithelial cells. J Biol Chem 286: $35755-35762$

Tollefsbol TO (2017) Handbook of epigenetics: the new molecular and medical genetics (2nd ed.). Academic Press, London

Vel Szic KS, Declerck K, Vidakovic M, Berghe WV (2015) From inflammaging to healthy aging by dietary lifestyle choices: is epigenetics the key to personalized nutrition? Clin Epigenetics 7:33

Wang Y, Yan L, Zhang Z, Prado E, Fu L, Xu X, Du L (2018) Epigenetic regulation and its therapeutic potential in pulmonary hypertension. Front Pharmacol 9:241

Wong CC, Qian Y, Yu J (2017) Interplay between epigenetics and metabolism in oncogenesis: mechanisms and therapeutic approaches. Oncogene 36:3359

Woo H, Ha SD, Lee SB, Buratowski S, Kim T (2017) Modulation of gene expression dynamics by co-transcriptional histone methylations. Exp Mol Med 49:326

Xia G, Schneider-Stock R, Diestel A, Habold C, Krueger S, Roessner A, Naumann M, Lendeckel U (2008) Helicobacter pylori regulates p21 WAF1 by histone $\mathrm{H} 4$ acetylation. Biochem Biophys Res Commun 369:526-531

Zhou M, He J, Shen Y, Zhang C, Wang J, Chen Y (2017) New frontiers in genetics, gut microbiota, and immunity: a Rosetta stone for the pathogenesis of inflammatory bowel disease. Biomed Res Int 2017:8201672 